\title{
A solid-state device for presenting moving shadows
}

NORMAN M. WEINBERGER and DAVID A. GOODMAN, DEPARTMENT OF PSYCHOBIOLOGY, UNIVERSITY OF CALIFORNIA, Irvine, California 92664

The use of luminescent panels controlled by solid-state circuitry to produce moving shadows is described. Advantages include lack of vibration, noise, and heat.

The problem of presenting an advancing or retreating shadow to an animal has ordinarily been solved by interrupting a constant source of illumination (e.g., light bulb) with a moving vane (see, e.g., Russell, 1967). However, the use of animals that are highly sensitive to changes in either temperature or vibration, or both, precludes the use of incandescent lamps and motor-driven shadow-casters. For studies of Necturus maculosus (mud puppy), a wholly aquatic amphibian, we have developed a heatless, vibrationless shadow apparatus. Additional requirements included ready $E$ control of shadow velocity and direction, and the possibility of producing a moving stripe in addition to a moving shadow.

\section{METHOD}

The major components of the present system consist of luminescent strips, manufactured by Sylvania as "Panelescent Tape-Lite," Series PTL 175. Electroluminescence produces light, but not heat, by exciting phosphors with an alternating current field. Brightness is a function of both voltage and frequency. The latter feature permits control of intensity by varying the frequency of an oscillator (between 50 and $1000 \mathrm{~Hz}$ ). We have been using standard line voltage $(110 \mathrm{~V})$ and frequency $(60 \mathrm{~Hz})$.

The shadow apparatus consists of six $13 \times 2 \frac{1}{2}$ in. Panelescent strips arranged side-by-side and affixed to a false ceiling over the animal's aquarium (Fig. 1). An apparent moving shadow is produced by sequentially switching off current to the strips. This is accomplished by solid-state logic modules (Digi-Bits) consisting of a multivibrator, flip-flops, and AND gates that control six reed relays, one for each strip. These modules simply count to six, activate the relays in sequence, and switch off the current supplied to each strip via the normally open contact of each relay. The circuit diagram is shown in Fig. 2 . The rate of switching, which determines the apparent velocity of the shadow, is controlled by the multivibrator, and can be changed easily to suit changing experimental conditions.

We have found a shadow of approximately $0.11 \mathrm{~m} / \mathrm{sec}$ to be very effective in eliciting interruption of gill beat and heart beat in Necturus, that is, evoking a defensive or orienting reflex. This is produced by sequential interruption of current for $0.6 \mathrm{sec}$ per strip. Placement of the luminescent strips $12 \mathrm{in}$. above the aquarium results in a moving shadow approximately $2 \frac{1}{2}$ in. in width at the level of the animal. Additional logic and switching circuits permit the sequence of current interruption to be

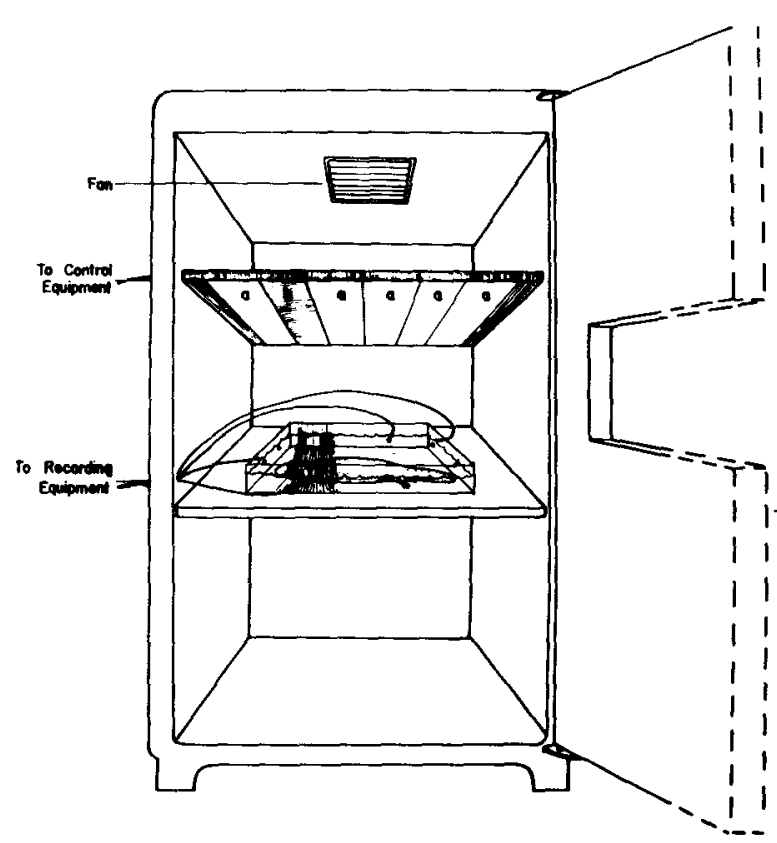

Fig. 1. Diagram of the shadow apparatus positioned above a Necturus (mud puppy). The a sections represent illuminated luminescent panels; $\boldsymbol{b}$ represents the unilluminated panel casting a shadow upon Necturus. Wires leading from the mud puppy's shallow acquarium to recording equipment carry output of photo-cells and aquatic electrodes which pick up EKG and gill movement. The entire apparatus is enclosed within a refrigerator kept at $19 \mathrm{deg}$.

reversed, thus reversing the direction of the moving shadow. Another option permits the strips to be turned off, so that sequentially switching on the current provides an apparent moving stripe across a dark background.

These luminescent strips are available in lengths of up to $150 \mathrm{ft}$, and may be cut to size without affecting performance. This is important because "white" strips, from different shipments, tend to vary, and have a slight blue, yellow, or green cast. Quality control can best be achieved in the lab by purchasing a long section and cutting it into lengths suitable for the particular experimental needs.

\section{REFERENCE}

RUSSELL, E. M. Changes in the behavior of Lebistes reticulatus upon a repeated shadow stimulus. Animal Behaviour, 1967, 15, 574-585.

NOTE

1. This research was supported by PHS Research Grant MH 11250 from the National Institute of Mental Health to N.M.W.

(See Fig. 2 on following page.) 


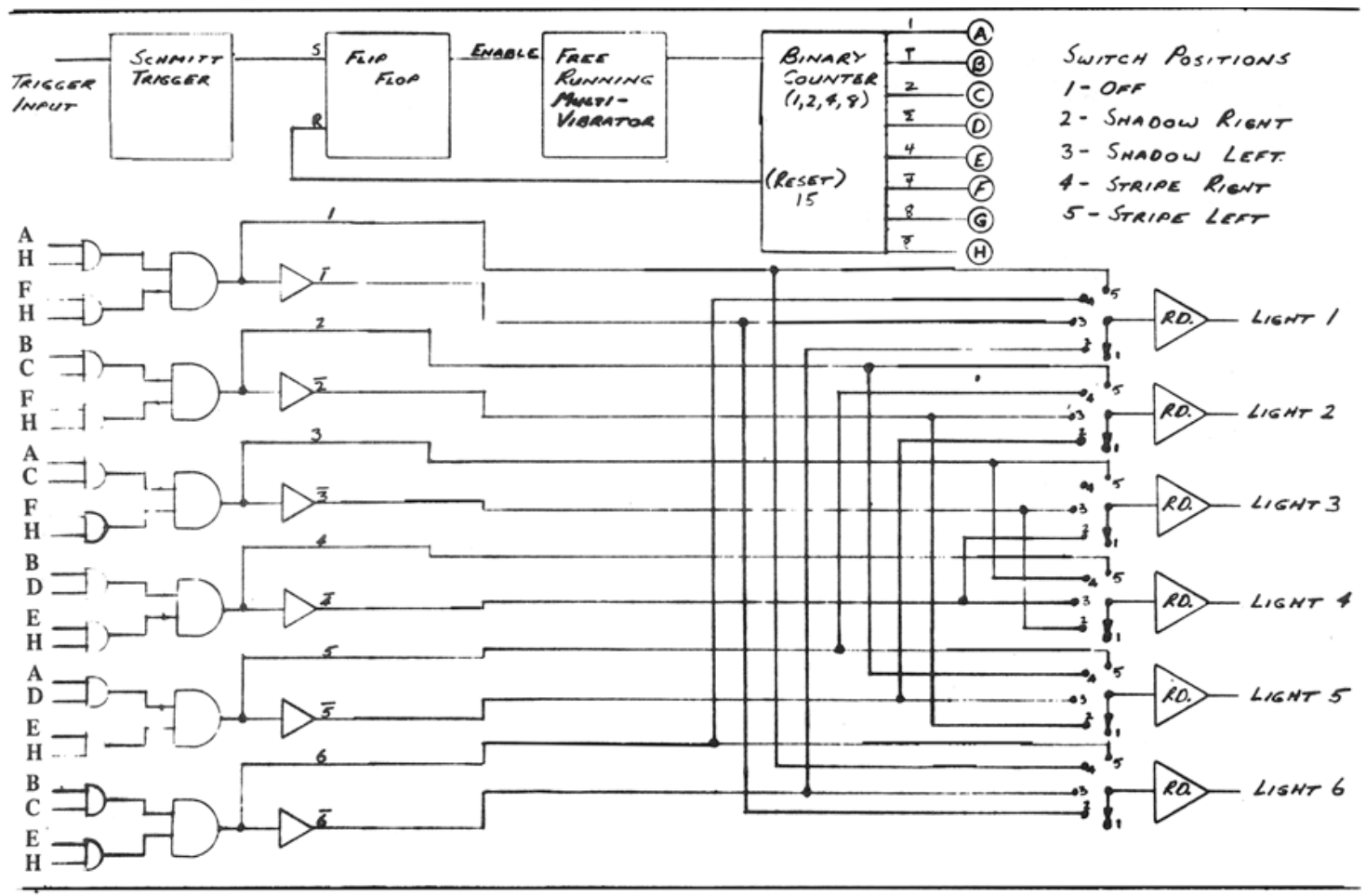

Fig. 2. Circuit diagram for sequentially switching six luminescent strips ("lights") on-off (stripe) or off-on (shadow) in either of two directions: left (Lights 1-6) or right (6-1). Solid-state devices perform all programming functions. A trigger input, from either an external device (e.g., switch closure) or other digital logic, initiates one sequence of stimulus presentation by triggering a Schmitt trigger that provides a standard pulse to a control flip-flop. This flip-flop is set into the "on" or " 1 " state, providing a continuous enable voltage to a variable multivibrator that emits regularly spaced pulses as a function of its rate setting. These timing pulses drive a binary counter, which consists of four flip-flops, whose output is decoded by an AND gate network to provide voltages for the decimal numbers 1 ...6. Inverters also provide not $1 \ldots$. . Switch position setting determines the sequences of energizing relay drivers (RD), left to right or vice versa, and whether the lamps are to be switched on (moving stripe) or off (moving shadow). The binary counter provides a rest pulse at the count of 15 to terminate the sequence by resetting the control flip-flop to the off or zero state. A four-bit binary counter is illustrated to allow easy expansion for more than six lamps. Two-legged AND gates are illustrated because some manufacturers (e.g., BRS Electronics, "Digi-Bits") do not supply four-legged gates. Six four-legged AND gates could replace those shown. As drawn, the circuit may be used with any digital logic modules provided that the relay or lamp drivers are appropriate for the lamp used. For switching $110 \mathrm{~V}$ ac, we use reed relays in place of relay drivers and switch one side of the standard 110.V line through the "common" and "normally open" points. 